\title{
A detectable antihelium flux from dark matter annihilation
}

\section{Martin Wolfgang Winkler ${ }^{a, *}$}

${ }^{a}$ The Oskar Klein Centre, Department of Physics, Stockholm University, AlbaNova, SE-10691 Stockholm, Sweden

E-mail: martin.winkler@su.se

We show that a previously neglected process - the decay of $\bar{\Lambda}_{b}$ baryons to antihelium - can greatly enhance the cosmic ray antihelium flux. Such $\bar{\Lambda}_{b}$ baryons frequently occur as intermediate states in dark matter annihilation into heavy quarks or gauge bosons. Due to their antibaryon number and $5.6 \mathrm{GeV}$ rest mass, they effectively decay to multi-antinucleon final states with small relative momentum which can bind into antihelium. We find that $\bar{\Lambda}_{b}$ decays increase the antihelium signal by up to two orders of magnitude and possibly even account for the tentative observation of $O(10)$ antihelium events at AMS-02.

$37^{\text {th }}$ International Cosmic Ray Conference (ICRC 2021)

July 12th - 23rd, 2021

Online - Berlin, Germany

${ }^{*}$ Presenter 


\section{Introduction}

Cosmic ray antinuclei fluxes play an important role in the indirect search for dark matter. While dark matter annihilations typically mostly induce $\gamma$-rays and light leptons, in rare cases antinucleons with small relative momentum form and bind together into an antinucleus. Excitingly, the astrophysical background of antinuclei stemming from the scattering of cosmic ray protons or helium on the interstellar matter carries a similar or even stronger suppression compared to the dark matter signal $[1,2]$. Therefore, the discovery of a single cosmic ray antinucleus could already provide evidence for dark matter annihilation [3,4].

A particularly promising target is the cosmic ray antideuteron flux. In the low-energy range the specificity of the dark matter signal is high and the flux is potentially detectable [4]. Significant satellite- [5] and balloon-based [6] efforts to detect these antideuterons are ongoing. In the case of antihelium, on the other hand, it seemed that neither dark matter annihilation nor astrophysics can lead to a flux within the sensitivity of present experiments [1, 7-13].

In this light, the tentative detection of $O(10)$ antihelium events at the Alpha Magnetic Spectrometer (AMS-02) [14, 15] appeared particularly surprising and has triggered speculations about more exotic sources like primordial antimatter clouds [11]. However, we realized [16] that the dark matter-induced antihelium flux could be orders of magnitude larger than previously estimated. The key observation is that intermediate $\bar{\Lambda}_{b}$ decays, which frequently occur in dark matter annihilation, can strongly boost the antihelium formation.

The $\bar{\Lambda}_{b}$-contribution, which has been entirely missed in the previous literature, can be decisive due to the $5.6 \mathrm{GeV}$ rest mass and the antibaryon number of $\bar{\Lambda}_{b}$. These two properties allow for a surprisingly high decay rate of $\bar{\Lambda}_{b}$ into three antinucleons with small relative momentum, which then efficiently merge into antihelium. Intriguingly, because the so-produced antinuclei inherit the Lorentz boost of the $\bar{\Lambda}_{b}$, they automatically obtain the large center-of-mass momenta necessary to fit the AMS-02 signal $[14,15]$.

\section{Calculation of Antinucleus Injection Spectra}

In order to determine antideuteron and antihelium spectra we employ a Monte Carlo approach and apply the event-by-event coalescence model [8, 9, 17-19]. Antinucleus formation requires the momenta of the antinucleons in their center-of-mass frame to be smaller than $p_{c} / 2$ (antideuteron) or $2^{1 / 6} p_{c} / 2$ (antihelium-3, antitriton), where $p_{c}$ denotes the coalescence momentum. ${ }^{1}$ Additionally, we require all antinucleons to descend from the same interaction vertex. In contrast to previous analyses $[8,9,12]$ which rejected all antinucleons from displaced vertices, our condition enables displaced antinucleus formation if all antinucleons come from the same displaced vertex. For this reason antihelium from $\bar{\Lambda}_{b}$ decays is correctly included in our analysis, while it has been missed previously.

Dark matter annihilations are simulated with the Monte Carlo generators Pythia (version 8.2) [20] and Herwig (version 7.2) [21]. In the default configuration Pythia underestimated the $\bar{\Lambda}_{b}$-production ratio by a factor of $\sim 3$ compared to LEP data $f\left(b \rightarrow \Lambda_{b}\right)=0.1_{-0.03}^{+0.04}[22,23]$.

${ }^{1}$ Our coalescence condition for antihelium differs by $2^{1 / 6}$ compared to [8]. We chose our convention such that the definition of $p_{c}$ agrees between the event-by-event and the analytic coalescence model (see [16]). 


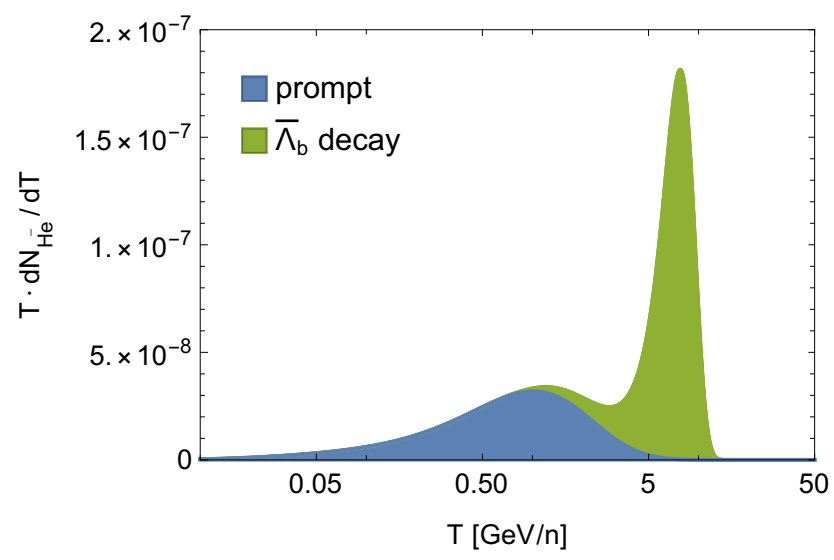

Figure 1: Antihelium injection spectrum (per annihilation) for $\chi \chi \rightarrow \bar{b} b$ with $m_{\chi}=67 \mathrm{GeV}$. The prompt and $\bar{\Lambda}_{b}$-induced contributions are shown separately.

Therefore we also consider a Pythia model (denoted " $\Lambda_{b}$-tune") with an increased diquark formation parameter (probQQtoQ) to match LEP data. ${ }^{2}$ Herwig offers the option to perform $\Lambda_{b}$ decays with the $B$-physics tool EvtGen, which we also consider. The choice of our four different Monte Carlo implementations allows us to access the relevant systematic uncertainties associated with antinucleus formation.

For the determination of $p_{c}$ we employ the antideuteron formation in Z-decays measured at ALEPH [26], and the antihelium spectra in $p p$-collisions at ALICE [27] (see [16]). The obtained antideuteron and antihelium coalescence momenta are listed separately for our four Monte Carlo configurations in Table 1.

\begin{tabular}{|ccccc|}
\hline channel & Pythia & Pythia $\bar{\Lambda}_{b}$-tune & Herwig & Herwig+EvtGen \\
\hline antideuteron & $215_{-23}^{+19} \mathrm{MeV}$ & $124_{-13}^{+11} \mathrm{MeV}$ & $201_{-24}^{+20} \mathrm{MeV}$ & $201_{-24}^{+20} \mathrm{MeV}$ \\
antihelium & $239_{-30}^{+25} \mathrm{MeV}$ & $138_{-16}^{+13} \mathrm{MeV}$ & $215_{-30}^{+25} \mathrm{MeV}$ & $215_{-30}^{+25} \mathrm{MeV}$ \\
\hline
\end{tabular}

Table 1: Coalescence momenta for antideuteron and antihelium formation.

We then calculate the antideuteron and antihelium injection spectra induced by dark matter annihilation. For a specific benchmark we choose a dark matter candidate with of $m_{\chi}=67 \mathrm{GeV}$ which annihilates into $\bar{b} b$ with a cross section of $\langle\sigma v\rangle=2 \cdot 10^{-26} \mathrm{~cm}^{3} \mathrm{~s}^{-1}$. This particular candidate is motivated by the galactic center gamma ray excess [28] and the antiproton excess [29]. Furthermore, a standard NFW profile with local density $0.38 \mathrm{GeV} \mathrm{cm}^{-3}$ is assumed [30].

We are especially interested in the antinucleus contribution induced by $\bar{\Lambda}_{b}$ decays which manifests at high energy since the antinucleus inherits the boost of the $\bar{\Lambda}_{b}$. In the case of antideuteron we observe that the prompt production at the initial vertex dominates. However, the displaced

\footnotetext{
2It was speculated that the increase of probQQtoQ which also enhances antinucleon production could lead to an overestimation of antihelium production in Pythia [24]. This is, however, not the case since we adjust antinucleus spectra to observed data through the choice of the coalescence momentum, i.e. the offset in the normalization of antinucleon spectra is effectively corrected for [25].
} 
component from $\bar{\Lambda}_{b}$ decays adds a high-energy peak to the spectrum which contributes between a few percent and one third to the total flux depending on the Monte Carlo implementation. ${ }^{3}$

In the case of antihelium the effect is even far more striking. In Figure 1 we depict the prompt and $\bar{\Lambda}_{b}$-induced antihelium injection spectrum in Pythia for the benchmark dark matter model. It can be seen that $\bar{\Lambda}_{b}$ baryons are responsible for two thirds of the antihelium nuclei and entirely dominate the high-energy spectrum. In the Pythia $\Lambda_{b}$-tune and in Herwig+EvtGen, the $\bar{\Lambda}_{b}$ contribution is even larger. Only in default Herwig antihelium from $\bar{\Lambda}_{b}$ is very suppressed since the cluster hadronization algorithm suppresses multi-antinucleon final states in the regime of a narrow phase space.

\section{Antideuteron Flux}

In order to calculate the resulting antinuclei fluxes, we employ a standard two-zone diffusion model fit to cosmic ray data [31-33]. Solar modulation is included through an improved force-field description [34].

The antideuteron flux for the benchmark dark matter model is shown in the left panel of Figure 2. We observe that $\bar{\Lambda}_{b}$ decays add an exciting new high-energy signature which can potentially be probed at AMS-02. While the $\bar{\Lambda}_{b}$-peak may occur slightly above the AMS-02 energy window for $m_{\chi}=67 \mathrm{GeV}$, it can easily be moved inside by considering a smaller dark matter mass.

\section{Antihelium Flux}

The antihelium flux (right panel of Figure 2) is entirely dominated by the $\bar{\Lambda}_{b}$-contribution for every model except the Herwig Monte Carlo implementation. As in the case of antideuterons, the new signature occurs at high energy $(T \sim 10 \mathrm{GeV} / \mathrm{n})$. Since the AMS-02 sensitivity is larger in this range the impact of $\bar{\Lambda}_{b}$ on the projected antihelium events is even stronger than on the integrated flux. In the default Pythia implementation 0.1 antihelium events per 10 years are predicted at AMS-02 compared to 0.007 without the $\bar{\Lambda}_{b}$ contribution. For Herwig+EvtGen and the Pythia $\Lambda_{b}$-tune, the antihelium signal is even enhanced by a factor $\sim 100$ and reaches 0.3 and 0.9 events respectively.

While the estimated dark matter signal is still somewhat smaller compared to the tentative observation of $O(10)$ antihelium events, the significant increase to antihelium production motivates the careful analysis of $\Lambda_{b}$ physics. Given the large variations in $\operatorname{Br}\left(\bar{\Lambda}_{b} \rightarrow \overline{\mathrm{He}}\right)$ between event generators, it is conceivable that the true antihelium flux even lies above the range obtained in this work. Moreover, several additional mechanisms (e.g., Alfvénic reacceleration [36]) could further enhance the antihelium flux. Finally, we find that a further increase of the antihelium events by a factor of a few can be achieved in dark matter models with light mediators which decay to $\bar{b} b$ [16].

\section{Conclusion and Outlook}

In contrast to previous work, we have shown that dark matter annihilations can produce a detectable antihelium flux. Our model does not invoke any exotic physics, rather it properly

\footnotetext{
${ }^{3}$ The decay of $B$-mesons also contributes to the high-energy peak.
} 

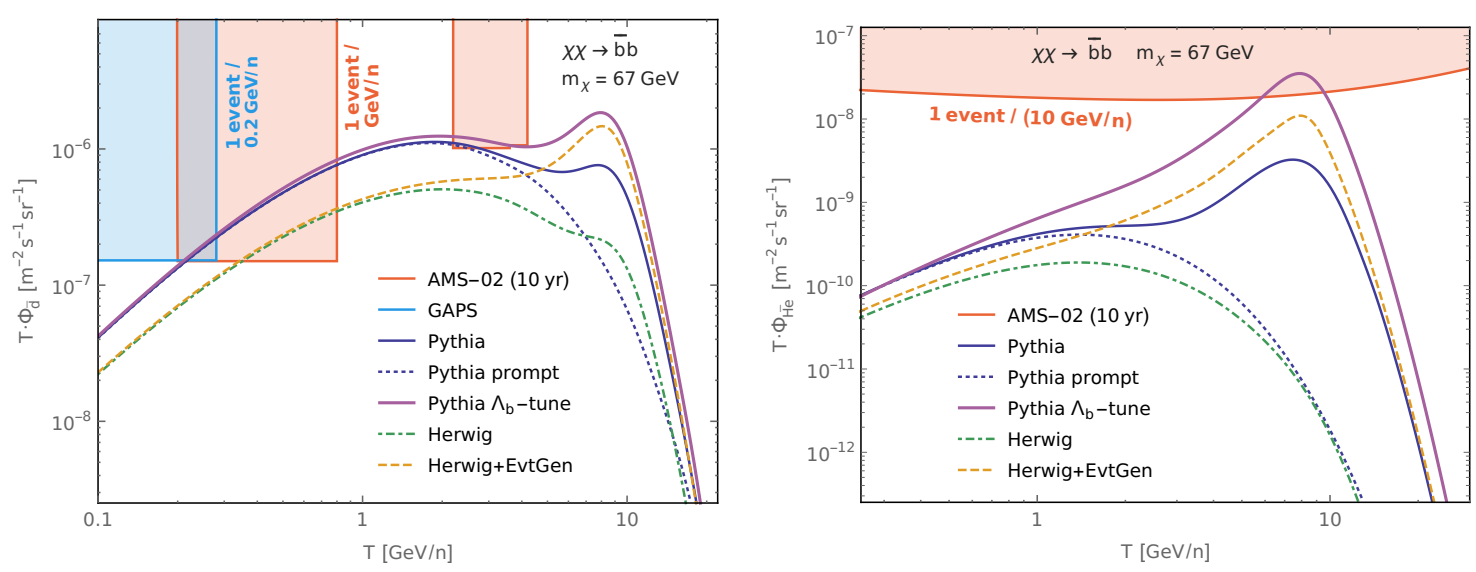

Figure 2: Antideuteron (left panel) and antihelium flux (right panel) from dark matter annihilation with $\langle\sigma v\rangle=2 \times 10^{-26} \mathrm{~cm}^{3} / s$ for several Monte Carlo implementations. The AMS-02 and GAPS antideuteron sensitivities [6] and the AMS-02 antihelium sensitivity [35] are also shown.

accounts for the antihelium formation from intermediate $\bar{\Lambda}_{b}$ decays. The $\bar{\Lambda}_{b}$ contribution to the antihelium flux entirely dominates the high-energy spectrum and increases the antihelium signal at AMS-02 by up to a factor $\sim 100$.

Our results are produced by leading algorithms such as Pythia and EvtGen. However, it is notable that standard Herwig analyses produce a negligible antihelium flux from $\bar{\Lambda}_{b}$ decay. While all other Monte Carlo implementations support a drastic enhancement of the antihelium flux through $\bar{\Lambda}_{b}$, the exact strength of the effect varies among them.

Our work, therefore, strongly motivates a dedicated experimental program to measure the branching ratio $\operatorname{Br}\left(\bar{\Lambda}_{b} \rightarrow \overline{\mathrm{He}}\right)$. From inclusive bottom production rates at the LHC [37] we can estimate an $O(0.1 \mathrm{nb})$ cross section for the process $p p \rightarrow \bar{\Lambda}_{b} \rightarrow \overline{\mathrm{He}}$ which is likely testable at the LHC. The prospect of connecting an antihelium signal at AMS-02 to an accelerator measurement of $\bar{\Lambda}_{b} \rightarrow \overline{\mathrm{He}}$ is extremely exciting.

\section{References}

[1] R. Duperray, B. Baret, D. Maurin, G. Boudoul, A. Barrau, L. Derome et al., Flux of light antimatter nuclei near Earth, induced by cosmic rays in the Galaxy and in the atmosphere, Phys. Rev. D 71 (2005) 083013 [astro-ph/0503544].

[2] D.-M. Gomez-Coral, A. Menchaca Rocha, V. Grabski, A. Datta, P. von Doetinchem and A. Shukla, Deuteron and Antideuteron Production Simulation in Cosmic-Ray Interactions, Phys. Rev. D 98 (2018) 023012 [1806.09303].

[3] F. Donato, N. Fornengo and P. Salati, Anti-deuterons as a signature of supersymmetric dark matter, Phys. Rev. D 62 (2000) 043003 [hep-ph/9904481].

[4] H. Baer and S. Profumo, Low energy antideuterons: shedding light on dark matter, JCAP 12 (2005) 008 [astro-ph/0510722]. 
[5] AMS 02 collaboration, The antimatter spectrometer (AMS-02): A particle physics detector in space, Nucl. Instrum. Meth. A 588 (2008) 227.

[6] GAPS collaboration, Antideuteron Sensitivity for the GAPS Experiment, Astropart. Phys. 74 (2016) 6 [1506. 02513].

[7] P. Chardonnet, J. Orloff and P. Salati, The Production of antimatter in our galaxy, Phys. Lett. B 409 (1997) 313 [astro-ph/9705110].

[8] E. Carlson, A. Coogan, T. Linden, S. Profumo, A. Ibarra and S. Wild, Antihelium from Dark Matter, Phys. Rev. D 89 (2014) 076005 [1401.2461].

[9] M. Cirelli, N. Fornengo, M. Taoso and A. Vittino, Anti-helium from Dark Matter annihilations, JHEP 08 (2014) 009 [1401. 4017].

[10] K. Blum, K.C.Y. Ng, R. Sato and M. Takimoto, Cosmic rays, antihelium, and an old navy spotlight, Phys. Rev. D 96 (2017) 103021 [1704.05431].

[11] V. Poulin, P. Salati, I. Cholis, M. Kamionkowski and J. Silk, Where do the AMS-02 antihelium events come from?, Phys. Rev. D 99 (2019) 023016 [1808.08961].

[12] M. Kachelriess, S. Ostapchenko and J. Tjemsland, Revisiting cosmic ray antinuclei fluxes with a new coalescence mode, 2002.10481.

[13] A. Shukla, A. Datta, P. von Doetinchem, D.-M. Gomez-Coral and C. Kanitz, Large-scale Simulations of Antihelium Production in Cosmic-ray Interactions, 2006.12707.

[14] S. Ting, The First Five Years of the Alpha Magnetic Spectrometer on the International Space Station, CERN Colloquium, 2016.

[15] AMS-02 collaboration, AMS Days at La Palma, La Palma, Canary Islands, Spain, 2018.

[16] M.W. Winkler and T. Linden, Dark Matter Annihilation Can Produce a Detectable Antihelium Flux through $\bar{\Lambda}_{b}$ Decays, Phys. Rev. Lett. 126 (2021) 101101 [2006.16251].

[17] M. Kadastik, M. Raidal and A. Strumia, Enhanced anti-deuteron Dark Matter signal and the implications of PAMELA, Phys. Lett. B 683 (2010) 248 [0908 . 1578].

[18] L. Dal and M. Kachelriess, Antideuterons from dark matter annihilations and hadronization model dependence, Phys. Rev. D 86 (2012) 103536 [1207.4560].

[19] N. Fornengo, L. Maccione and A. Vittino, Dark matter searches with cosmic antideuterons: status and perspectives, JCAP 09 (2013) 031 [1306.4171].

[20] T. Sjöstrand, S. Ask, J.R. Christiansen, R. Corke, N. Desai, P. Ilten et al., An introduction to PYTHIA 8.2, Comput. Phys. Commun. 191 (2015) 159 [1410.3012].

[21] J. Bellm et al., Herwig 7.0/Herwig++ 3.0 release note, Eur. Phys. J. C 76 (2016) 196 [1512.01178]. 
[22] Particle Data Group collaboration, Review of particle physics. Particle Data Group, Eur. Phys. J. C 3 (1998) 1.

[23] OPAL collaboration, A Measurement of the product branching ratio $f(b \rightarrow \operatorname{Lambda}(b)) x$ BR(Lambda(b) $\rightarrow$ Lambda X) in Z0 decays, Eur. Phys. J. C 9 (1999) 1 [hep-ex/9812018].

[24] M. Kachelriess, S. Ostapchenko and J. Tjemsland, Comment on "Dark Matter Annihilation Can Produce a Detectable Antihelium Flux through $\bar{\Lambda}_{b}$ Decays”, 2105.00799.

[25] M.W. Winkler and T. Linden, Response to Comment on "Dark Matter Annihilation Can Produce a Detectable Antihelium Flux through $\bar{\Lambda}_{b}$ Decays", 2106.00053.

[26] ALEPH collaboration, Deuteron and anti-deuteron production in $e+e$-collisions at the $Z$ resonance, Phys. Lett. B639 (2006) 192 [hep-ex/0604023].

[27] ALICE collaboration, Production of deuterons, tritons, ${ }^{3}$ He nuclei and their antinuclei in pp collisions at $\sqrt{s}=0.9,2.76$ and 7 TeV, Phys. Rev. C97 (2018) 024615 [1709.08522].

[28] L. Goodenough and D. Hooper, Possible Evidence For Dark Matter Annihilation In The Inner Milky Way From The Fermi Gamma Ray Space Telescope, 0910 . 2998.

[29] A. Cuoco, M. Krämer and M. Korsmeier, Novel Dark Matter Constraints from Antiprotons in Light of AMS-02, Phys. Rev. Lett. 118 (2017) 191102 [1610.03071].

[30] P. de Salas, K. Malhan, K. Freese, K. Hattori and M. Valluri, On the estimation of the Local Dark Matter Density using the rotation curve of the Milky Way, JCAP 10 (2019) 037 [1906.06133].

[31] A. Reinert and M.W. Winkler, A Precision Search for WIMPs with Charged Cosmic Rays, JCAP 01 (2018) 055 [1712.00002].

[32] J. Heisig, M. Korsmeier and M.W. Winkler, Dark matter or correlated errors: Systematics of the AMS-02 antiproton excess, Phys. Rev. Res. 2 (2020) 043017 [2005 . 04237].

[33] M. Di Mauro and M.W. Winkler, Multimessenger constraints on the dark matter interpretation of the Fermi-LAT Galactic center excess, Phys. Rev. D 103 (2021) 123005 [2101.11027].

[34] I. Cholis, D. Hooper and T. Linden, A Predictive Analytic Model for the Solar Modulation of Cosmic Rays, Phys. Rev. D 93 (2016) 043016 [1511.01507].

[35] A. Kounine, AMS Experiment on the International Space Station, Talk at ICRC 2011 (2011) .

[36] I. Cholis, T. Linden and D. Hooper, Anti-Deuterons and Anti-Helium Nuclei from Annihilating Dark Matter, 2001.08749.

[37] CMS collaboration, Inclusive b-jet production in pp collisions at $\sqrt{s}=7 \mathrm{TeV}, \mathrm{JHEP} 04$ (2012) 084 [1202.4617]. 